\title{
Influence de la sécheresse sur la croissance et la photosynthèse du cèdre
}

\author{
G. AUSSENAC, D. FINKELSTEIN \\ I.N.R.A., Station de Sylviculture et de Production \\ Centre de Recherches de Nancy \\ Champenoux, F 54280 Seichamps
}

\begin{abstract}
Résumé
L'étude des seuils de sécheresse influençant la croissance et la photosynthèse a été entreprise chez le cèdre de différentes origines. Les résultats obtenus font apparaître des différences très nettes dans les niveaux de sécheresse affectant respectivement la croissance et la photosynthèse.

La croissance en hauteur est freinée lorsque le potenticl de base des plants atteint - 13 bars et elle devient nulle atu-delà de -21 bars.

D’une ficçon générale, la photosynthèse se poursuit à des niveaux de sécheresse plus importants que la croissance en hauteur. Ainsi, dans le cas de la provenance Ventoux lorsqu'intervient l'arrêt de la croissance, la photosynthèse représente encore 55 p. 100 du taux maximum mesuré. Les provenances méditerranéennes étudiées (Ventoux, Lubéron, Sidi-Ifri, Paphos, Sutlegen) cessent leur assimilation photosynthétique entre -50 et - 60 bars, alors que clans le cas de Cedrus deodara, le niveau correspondant est de - 30 bars.

Une comparaison avec les résultats obtenus pour d'autres résineux montre que le cèdre est l'essence dont l'activité photosynthétique peut se poursuivre aux niveaux de sécheresse les plus importants.
\end{abstract}

\section{Introduction}

Dans les régions méridionales de la France, le cèdre est maintenant utilisé pour la reconstitution de la forêt méditerranéenne. Mais la généralisation de son utilisation en reboisement suppose une amélioration de la connaissance de son comportement écophysiologique en particulier vis-à-vis de la sécheresse qui constitue le principal facteur limitant de la végétation forestière dans cette région. Malheureusement, dans ce domaine, il existe très peu de données disponibles. Cependant un certain nombre de travaux : Aussenac \& Granier, 1978, Pradal, 1979, Finkelstein, 1981, Aussenac \& VAletTE, 1982, ont récemment apporté d'utiles informations sur cette essence.

Le but du présent travail est d'étudier les seuils de sécheresse influençant la croissance et la photosynthèse du cèdre afin de préciser son comportement. Il s'agit aussi 
de mettre en évidence d'éventuelles différences entre diverses origines : Maroc, France, Chypre, Turquie, Inde et d'essayer de situer cette essence par rapport à d'autres résineux.

\section{Matériel et méthodes}

Deux séries de mesures ont été effectuées : dans la première on a étudié en serre la croissance en hauteur de cèdres de la provenance Ventoux, en relation avec le dessèchement du sol; dans la deuxième c'est l'activité photosynthétique de six provenances différentes qui a été étudiée en relation avec leur état hydrique en phase de dessèchement.

2.1. Effet du stress hydrique sur la croissance (première expérimentation)

\subsection{Origine des plants}

L'expérience a été réalisée sur 180 plants de Cedrus atlantica Manetti de la provenance Mont Ventoux (Vaucluse). Les plants provenant de la pépinière du Ruscas (Var) (1) ont été repiqués début mars 1976 à l’âge de 4 ans dans des pots en plastique d'une contenance de 9 litres. Le substrat utilisé provient d'un horizon A des sols bruns lessivés de texture limono-argileuse de la forêt domaniale d'Amance (Meurthe-etMoselle). Fin 1976 les plants avaient une hauteur moyenne de $577 \mathrm{~mm}$.

\subsection{Protocole d'alimentation hydrique}

L'expérimentation a été réalisée en 1977, trois modalités ont été définies (60 plants par modalité) :

- modalité humide : arbres maintenus à la capacité au champ (humidité pondérale de 32 p. 100, pF 2,4);

- modalité sèche : arbres maintenus à un niveau moyen de sécheresse (humidité pondérale de 16 p. 100, pF 3,6);

- modalité alternative : arbres subissant des cycles de dessèchement allant jusqu'à $\mathrm{pF}=3,6$ avec ensuite réhydratation jusqu'à la capacité au champ. Dans les faits, compte tenu du rythme de contrôle de l'humidité du sol, le pF a selon les cas largement dépassé 3,6.

Connaissant le poids de terre sèche et les divers accessoires pour chacun des plants, une simple pesée (portée $13 \mathrm{~kg}$, précision $1 \mathrm{~g}$ ) permet de calculcr l'humidité pondérale du sol. Le potentiel hydrique correspondant w sol est obtenu à partir de la courbe pF/humidité pondérale établie pour la terre utilisée. La mesure a été effectuée au laboratoire en utilisant une presse à membrane. Les pots sont pesés deux fois par semaine et si besoin est, ils sont arrosés jusqu'au niveau d'humidité du sol désiré.

La croissance en hauteur de la pousse terminale des plants est mesurée deux fois par semaine; les mesures sont effectuées à l'aide d'une règle graduée en millimètres (précision $\pm 1 \mathrm{~mm}$ ).

(1) Ces plants ont été fournis par la station de Sylviculture méditerranéenne d'Avignon. 
Le potentiel hydrique des plants est déterminé sur un rameau par la méthode de la bombe à pression (Scholander et al., 1965). La pression de sève mesurée en fin de nuit (appelée alors potentiel de base : $P_{n}$ ) est un point caractéristique de la cinétique d'évolution journalière du potentiel hydrique (AUSSENAC \& GRANiER, 1978). Hinckiey \& Ritchie (1973) définissent ce point comme le potentiel d'équilibre entre l'arbre et le sol lorsque la transpiration est faible ou nulle.

Au cours de quatre journées de septembre 1977, des mesures de potentiel de base ont été entreprises sur l'ensemble des plants de la modalité alternative. Le choix de cette modalité est dû à sa nature même qui entraîne, à un instant donné, une grande variabilité entre individus vis-à-vis de l'état hydrique des plants. On a pu de cette façon appréhender, en un minimum de temps, une gamme étendue de potentiels de base.

Dans le même temps, une pesée de chacun des pots est effectuée afin de déterminer l'humidité pondérale (et par voie de conséquence le potenticl hydrique) du sol au moment de la mesure. Une corrélation entre potentiel hydrique du sol et potentiel de base des plants a pu être mise en évidence (fig. 1); jusqu'à un potentiel hydrique du sol égal à $-11,5$ bars la relation suivante a été retenue : $\psi$ arbre $=1,941$ ( $\psi$ sol) $-3,989$ avec $\mathrm{r}^{2}=0,832$, pour un potentiel hydrique du sol inférieur à $-11,5$ bars le potentiel de base des plants se stabilise et la valeur moyenne de - 26,5 bars a été adoptée pour $\psi$ ' arbre ; ces relations ont permis, à partir des pesées bi-hebdomadaires, de suivre l'évolution saisonnière du potentiel de base et son effet sur la croissance.

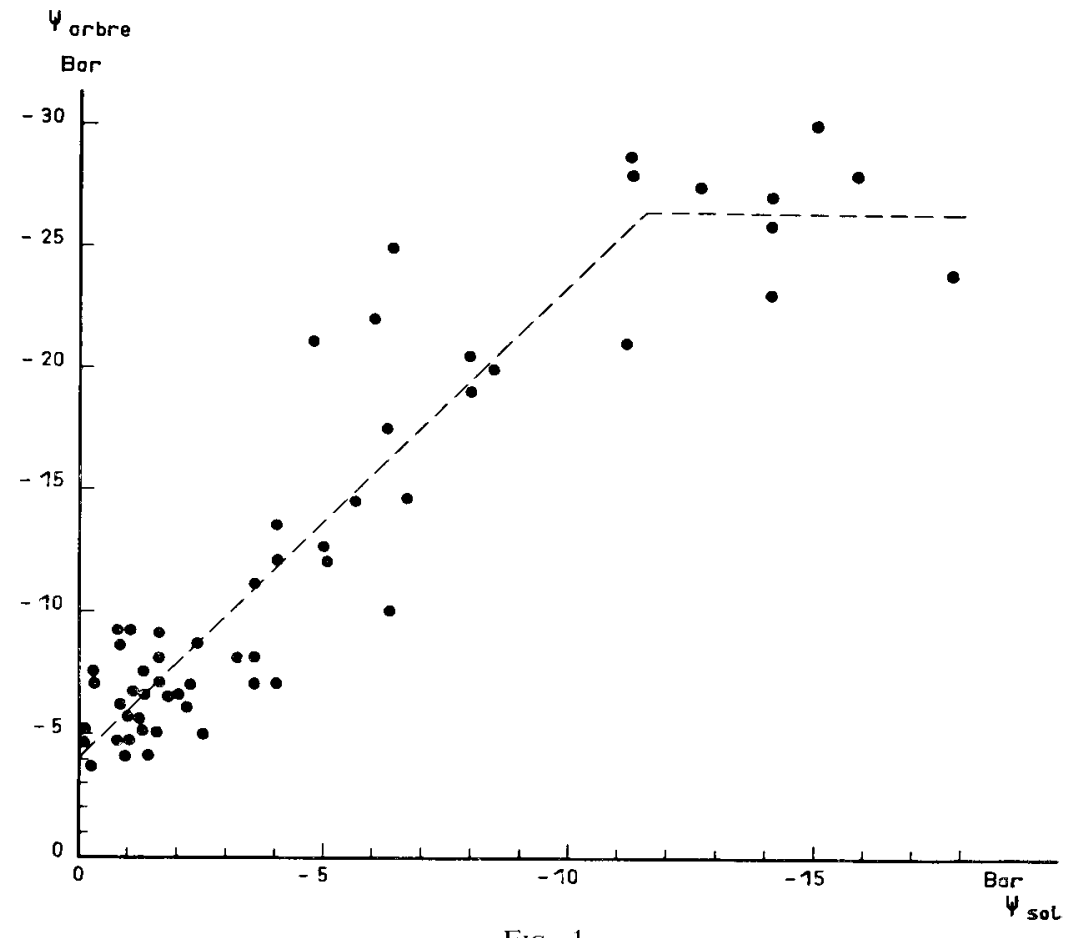

FiG. 1

Relation entre le potentiel hydrique du sol et le potentiel de base du plant. Relationship between soil water potential and predawn potential. 
2.2. Effet du stress hydricue sur la photosynthèse (deuxième expérimentation)

\subsection{Origine des plants}

Cette expérience a porté sur des plants de différentes origines (6 provenances) (tabl. 1) '11. Le substrat utilisé est un mélange sable + tourbe.

\section{Tableau 1}

Caractéristiques des plants utilisés pour la mesure de photosynthèse.

Characteristics of plants utilized in the study of photosynthesis.

\begin{tabular}{|c|c|c|c|c|}
\hline Espèce & Origine & Provenance & Age & Présentation \\
\hline C. atlantica $\ldots \ldots$ & France (Vaucluse) & Ventoux (1) & 2 ans & $\begin{array}{l}\text { Godet cartonné } \\
\text { individuel }\end{array}$ \\
\hline C. atlantica & France (Vaucluse) & Lubéron (1) & 2 ans & $\begin{array}{l}\text { Godet cartonné } \\
\text { individuel }\end{array}$ \\
\hline C. atlantica..... & Maroc & Sidi-Ifri & 2 ans & $\begin{array}{l}\text { Godet cartonné } \\
\text { individuel }\end{array}$ \\
\hline C. libani & Turquie & Sutlegen & 2 ans & $\begin{array}{l}\text { Godet cartonné } \\
\text { individuel }\end{array}$ \\
\hline C. brevifolia ...... & Chypre & Paphos & 2 ans & $\begin{array}{l}\text { Godet cartonné } \\
\text { individuel }\end{array}$ \\
\hline C. deodara .. & Inde & Himalaya & 2 ans & $\begin{array}{l}\text { Godet cartonné } \\
\text { individuel }\end{array}$ \\
\hline
\end{tabular}

(1) Peuplement artificiel d'origine inconnue.

\subsection{Protocole de dessèchement of de mesure}

Les plants (40 par provenance) sont placés dans une pièce climatiséc à proximité du laboratoire. Ils sont soumis au dessèchement du sol par simple arrêt d'arrosage.

Les températures et humidités relatives sont de l'ordre de 30 " $\mathrm{C}$ et 40 p. 100 durant la période de jour et de $24^{\circ} \mathrm{C}$ et $80 \mathrm{p}$. 100 durant la période nocturne. L'éclairage est assuré par quatre lampes à vapeur de mercure d'une puissance unitaire de 400 watts et par une lampe à vapeur de mercure haute pression de 2000 watts (surface du dispositif : $8 \mathrm{~m}^{2}$ ). L'éclairement moyen au niveau des plants est de l'ordre de 7000 lux. Un dispositif tournant des provenances est de plus prévu pour ne pas créer de biais systématique suivant la place occupée dans la pièce. L'allumage et l'extinction des lampes sont commandés par un système d'horloge électrique. La période diurne a une durée de 12 heures et débute à 11 heures du matin. De cette manière

(1) Ces plants ont été fournis par le Laboratoire d'Amélioration des arbres foresticrs de Bordeaux. Ils proviennent de la pépinière du Ruscas (Var). 
il est aisé de mesurer chaque matin le potenticl de base du plant à analyser dans la journéc. La mesure est effectuce sur un rameau de faible dimension afin de ne pas trop changer la surface foliaire du plant. Celui-ci est introduit dans une chambre dassimilation de type Sirigor-Siemens dans laquelle sont régulées la teneur en $\mathrm{CO}$. de laair, l'humidité et la température.

Le taux de CO.. à l'entréc et à la sortie de la chambre sont mesurés en différentiel à l'aide d'un analyseur de gaz par absorption infra-rouge (HARTMANN \& BRaUn, type Uras 2). La mesure s’effectue à une teneur en $\mathrm{CO}_{2 .}$ de $300 \mathrm{vpm}(0,03$ p. 100 vol.) à $20^{\circ} \mathrm{C}, 75$ p. 100 d'humidité relative et en éclairement saturant $\left(570, \mathrm{E}, \mathrm{m}^{-2} \mathrm{~s}^{-1}\right.$ pour le cèdre Finkelstein, 1981). La surface foliaire est estimée par planimétric à partir d'une photographic-contact des aiguilles (Kodabrome II RC F5) qui fournit la surface projetée par les aiguilles sur un plan. Après étude de la forme générale des sections d'aiguilles du cèdre, ces dernières ont été assimilées à un cylindre et la surface totale est obtenue en multipliant la surface projetée par $\pi$.

\section{Résultats}

\subsection{Effet du stress hydrique sur la croissance}

\subsection{Evolution saisomiere du potenticl de base}

Un plant, choisi en raison de sa taille moyenne, a été pris ici en exemple. L Cévolution de son potentiel de base ainsi que la croissance journalière moyenne par période sont portées sur la figure 2.

Deux périodes de croissance type peuvent être considérées. Du débourrement au 31 mai, période de croissance maximale, les valeurs de la croissance oscillent autour d'une valeur moyenne sensiblement constante et ses fluctuations sont exactement calquées sur celles du potentiel de base. Du $1^{\mathrm{m}}$ au 20 juin, on note toujours ce parallélisme, mais il s'y surimpose le rythme saisonnier de la croissance qui conduit à des croissances maximales journalières de plus en plus faibles juqu’à l’arrêt total.

\subsection{Détermination d'un seuil hydrique d'arrêt de la croissance en hautèr}

Les considérations précédentes sur l'évolution parallèle du potentiel de base et de l'élongation nous ont amenés à déterminer un seuil au-delà duquel la croissance est affectée par le déficit hydrique.

Cette recherche siest faite uniquement durant la période de croissance maximale (du débourrement jusqu'au $31 \mathrm{mai}$ ), pendant laquelle l'influence du stress hydrique n'est pas atténuée par la chute générale d'activité de fin de croissance. Nous avons alors noté, par plant et par période de mesure. les valeurs moyennes du potentiel de base et de la croissance journalière. Du fait de la variabilité de la croissance parmi les plants, le dernier paramètre a été rapporté en p. 100 de la valeur maximale atteinte par chaque individu.

La figure ainsi obtenue (fig. 3) se présente comme un nuage de points dont la courbe enveloppe est constituée de ceux pour lesquels seul le paramètre hydrique 


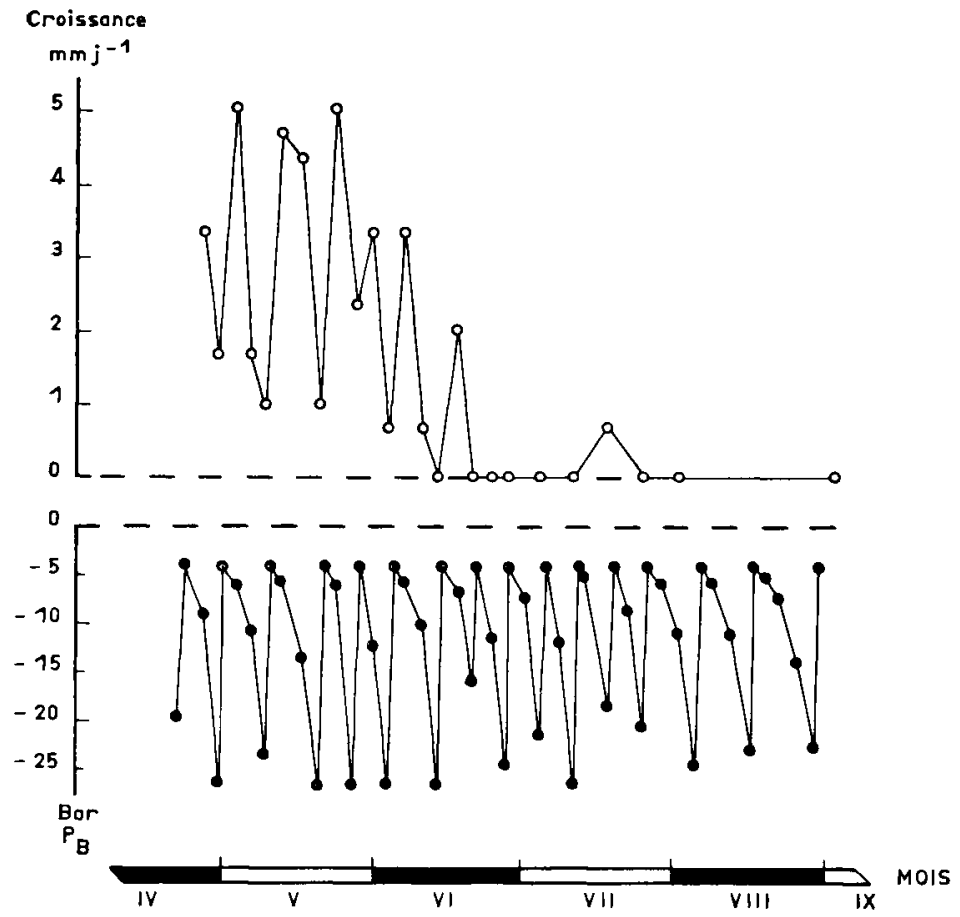

FIG. 2

Exemple d'évolution du potentiel de base $\left(P_{B}\right)$ et de la croissance journalière pour un plant de la modalité alternative humide-sec.

Example of predan'n potential evolution and daily growth for a plant in alternative situation humid-dry.

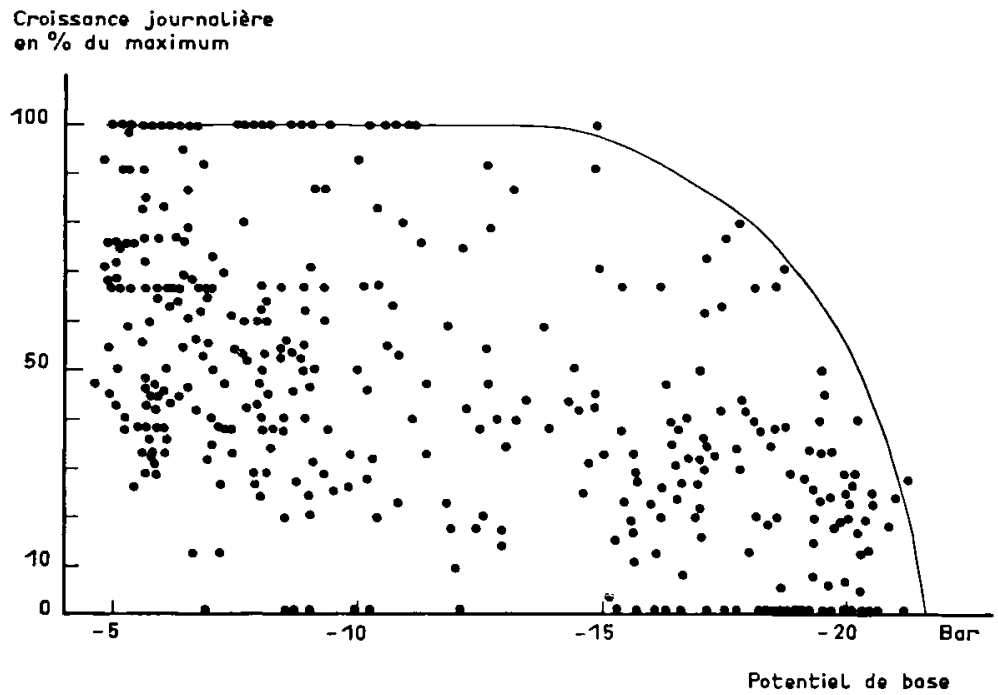

Fig. 3

Influence du potentiel de base sur la croissance. Influence of predawn potential on growth. 
conditionne les variations. Tous les autres points sont en deçà de la courbe enveloppe par le fait d'un facteur limitant autre que le potentiel de base (rayonnement, température, etc.). Interprétée de cette manière, la figure 3 montre alors que la croissance est affectée à partir d'un potentiel de base situé entre -13 et -15 bars et devient nulle pour une valeur de -21 bars.

\subsection{Effet du stress hydrique sur la photosynthèse}

En l'absence du stress hydrique, les taux de photosynthèse maximaux obtenus varient de $6,0 \mathrm{mg} \mathrm{CO}_{2} \mathrm{~h}^{-1} \mathrm{dm}^{-2}$ pour la provenance Himalaya à $15,1 \mathrm{mg} \mathrm{CO}_{2}$ $\mathrm{h}^{-1} \mathrm{dm}^{-2}$ pour Paphos.

Dans leur ensemble, les cèdres du pourtour méditerranéen $(C$. atlantica, $C$. libani, C. brevifolia) photosynthétisent jusqu'à des potentiels de base très bas (fig. 4). Cette activité chute dès l'apparition de la sécheresse ( -10 bars), mais peut se poursuivre faiblement au-delà de -50 bars. Cedrus deodara (Himalaya) présente des possibilités plus limitées : sa photosynthèse chute plus rapidement aux faibles déficits et devient négligeable au-delà de -35 bars.

Un examen plus détaillé des résultats montre que le taux de photosynthèse évolue différemment selon les provenances. Afin de simplifier l'analyse, 4 seuils de comparaison de la photosynthèse ont été déterminés : $-10,-20,-40$ et - 60 bars (tabl. 2). Les valeurs portées sur ce tableau correspondent aux courbes moyennes de la figure 4. Elles sont exprimées en p. 100 de la valeur maximale obtenue pour chaque provenance, permettant ainsi de comparer leur degré d'adaptation (à cet égard) au dessèchement du sol. Ainsi, quel que soit le potentiel de base, Cedrus deodara est la provenance la plus affectée par la sécheresse. A l'opposé, la provenance Sutlegen de Cedrus libani présente le meilleur comportement sur toute la gamme de potentiel. Il est également intéressant de noter que la photosynthèse de la provenance française Lubéron est bcaucoup plus touchée par le développement du stress hydrique que celle de la provenance Ventoux. Cette divergence a d'autant plus d'intérêt que les stations d'origine sont proches géographiquement.

\section{TABleau 2}

Photosynthèse (en p. 100 du taux maximum) obtenu à différents niveaux de stress hydrique.

Photosynthesis as a percentage of its maximum for different levels of plants moisture stress.

\begin{tabular}{|c|c|c|c|c|}
\hline \multirow{2}{*}{ Provenances } & \multicolumn{4}{|c|}{ \% de photosynthèse au seuil de } \\
\hline & -10 bars & -20 bars & -40 bars & -50 bars \\
\hline Himalaya & 57 & 23 & 5 & 0 \\
\hline Lubéron $\ldots \ldots \ldots \ldots \ldots$ & 57 & 38 & 19 & 15 \\
\hline Paphos $\ldots \ldots \ldots \ldots$ & 66 & 36 & 16 & 9 \\
\hline Sidi Ifri $\ldots \ldots \ldots \ldots$ & 80 & 45 & 17 & 11 \\
\hline Ventoux $\ldots \ldots \ldots \ldots$ & 91 & 58 & 24 & 15 \\
\hline Sutlegen $\ldots \ldots \ldots \ldots$ & 89 & 83 & 32 & 20 \\
\hline
\end{tabular}




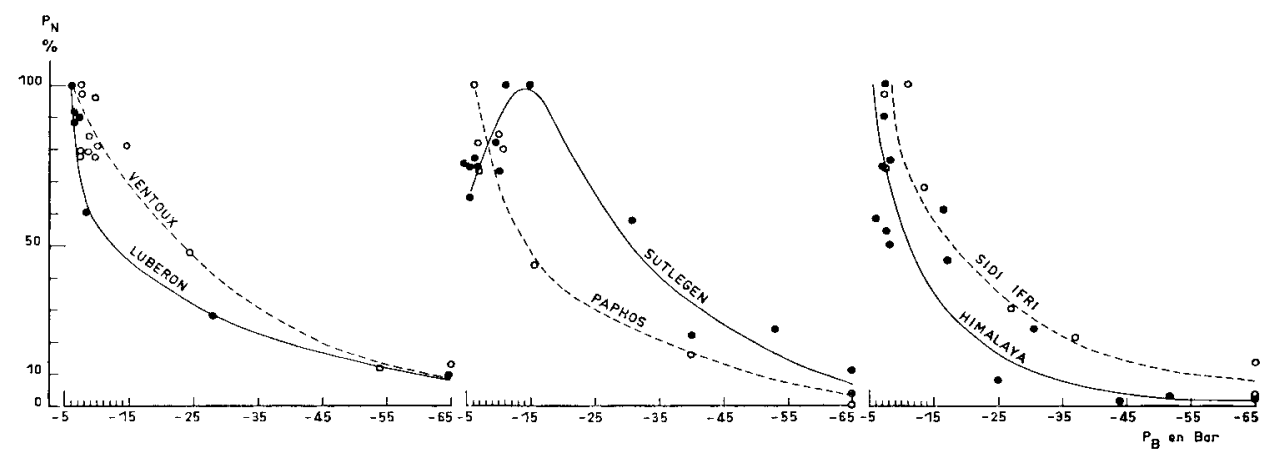

FIG. 4

Evolution de la photosynthèse nette $\left(P_{*}\right)$ (en pourcentage du maximum) en fonction du potentiel de base $\left(\boldsymbol{P}_{B}\right)$.

Net photosynthesis $\left(P_{N}\right)$ (percentage of maximum) as a fonction of the predawn potential $\left(P_{B}\right)$.

\begin{abstract}
Ventoux : 100 p. $100 \mathrm{P}_{\mathrm{x}}=7,2 \mathrm{mg} \mathrm{CO} \mathrm{h}^{-1} \mathrm{dm}^{2}$,
Lubéron : 100 p. $100 \mathrm{P}_{\mathrm{N}}=7,9 \mathrm{mg} \mathrm{CO}_{2} \mathrm{~h}^{-1} \mathrm{dm}^{-2}$,

Sutlegen : 100 p. $100 \mathrm{P}_{\mathrm{N}}=8,8 \mathrm{mg} \mathrm{CO} \mathrm{h}^{-1} \mathrm{dm}^{-2}$,

Paphos : 100 p. $100 \mathrm{P}_{\mathrm{N}}=15,1 \mathrm{mg} \mathrm{CO} \mathrm{h}^{-1} \mathrm{dm}^{-2}$,

Sidi Ifri : 100 p. $100 \mathrm{P}_{\mathrm{v}}=11,9 \mathrm{mg} \mathrm{CO} \mathrm{h}^{-1} \mathrm{dm}^{2}$,

Himalaya : 100 p. $100 \mathrm{P}_{\mathrm{x}}=6,0 \mathrm{mg} \mathrm{CO} \mathrm{h}^{-1} \mathrm{dm}^{-2}$.
\end{abstract}

\title{
4. Conclusion - Discussion
}

Les résultats obtenus font apparaître des différences très nettes dans les niveaux de sécheresse correspondant à l'arrêt de la croissance et de la photosynthèse.

Pour des plants de Cedrus atlantica Manetti âgés de 5 ans, la croissance en hauteur commence à être freinée lorsque le potentiel de base atteint -13 bars et elle est bloquée à -21 bars, ce qui correspond respectivement (fig. 1) à des potentiels du sol de - 4,6 bars et de $-8,7$ bars. Peu de données existent à ce sujet, néanmoins Glerum \& Pierpoint (1968) estiment à - 15 bars la tension de l'eau du sol nécessaire à l'arrêt de la croissance chez Pinus resinosa et Larix laricina alors que STransky \& Wilson (1964) avancent le chiffre de -25 bars pour Pinus taeda et Pinus echinata. Des différences dans les modalités d'établissement de la sécheresse pourraient expliquer des écarts aussi importants.

En conditions naturelles, au Mont Ventoux (Vaucluse) Aussenac \& Valette (1981) ont étudié l'évolution saisonnière de l'état hydrique de cèdres âgés de 17 à 25 ans. Ces auteurs ont trouvé que le potentiel de base pouvait atteindre, selon les stations, des valeurs inférieures à -21 bars durant une période de plus de 2 mois. Or, la croissance en hauteur du cèdre étant susceptible de s'étaler sur 5 mois, de mai à fin septembre (Aussenac, Granier, Gross, 1981), l'intervention de la sécheresse durant les mois d'été peut donc réduire de façon notable la taille de la pousse 
annuelle. Cependant, Finkelstein (1981) a montré pour le cèdre, que lorsque l'année sèche est suivie d’une année humide, il en résulte dans ce cas une stimulation de la croissance. L'influence immédiate de la sécheresse sur la croissance en hauteur différencie nettement le cèdre des espèces à pousse monocyclique courte chez lesquelles les effets de la sécheresse ne n'observent que l'année suivant son application.

D’une façon générale, la photosynthèse se poursuit à des niveaux de sécheresse plus importants que la croissance en hauteur. Ainsi, dans le cas de la provenance Ventoux, lorsqu'intervient l'arrêt de la croissance ( -21 bars), la photosynthèse représente encore 55 p. 100 du taux maximum mesuré.

\section{TABI.EAU 3}

Potentiel hydrique correspondant au seuil de $10 \mathrm{p} .100$ de la photosynthess maximale chez le cèdre et divers conifères.

Plant water potential at $10 \mathrm{p} .100$ of maximum photosynthesis for the Cedre and various conifers.

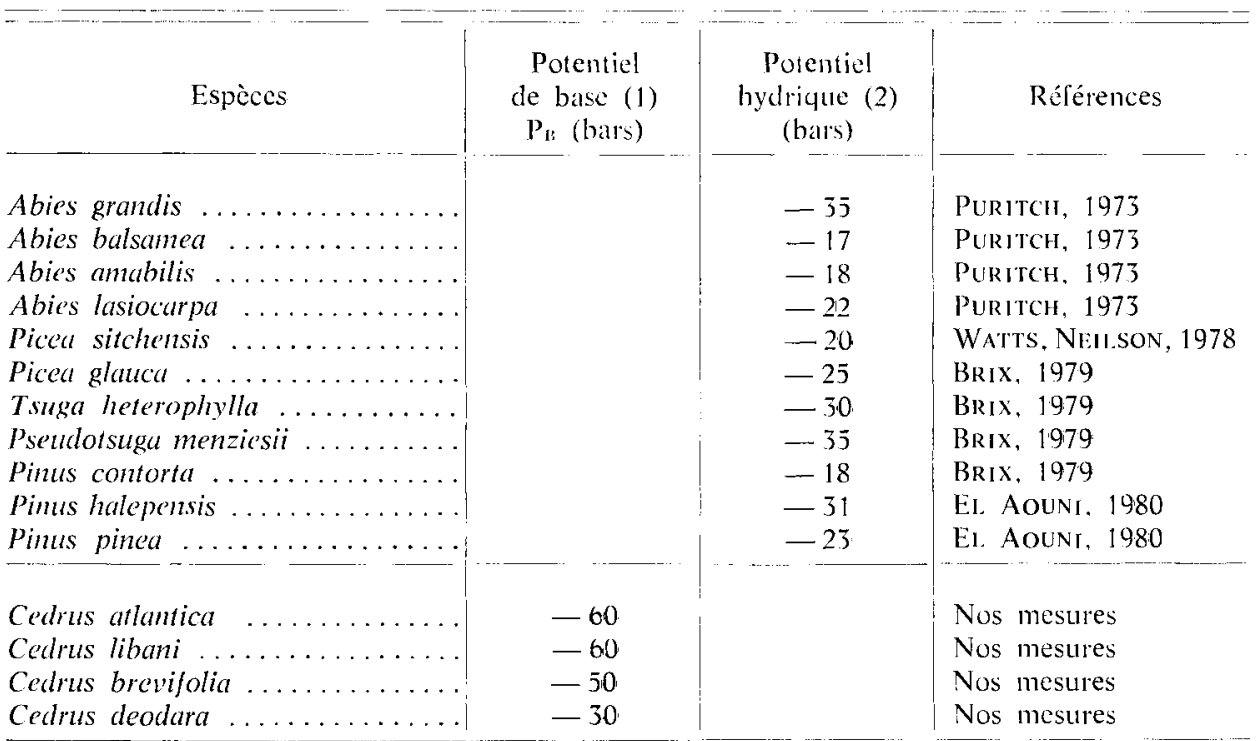

(1) Mesuré en fin de nuit avant la mesure de photosynthèse.

(2) Mesuré au moment de la mesure de photosynthèse.

En ce qui concerne larrêt de la photosynthèse, compte tenu de la dispersion des points de mesure, il nous semble intéressant de prendre comme référence de photosynthèse négligeable, le seuil de 10 p. 100 du taux maximum. A cet égard, on peut considérer que les provenances méditerranéennes étudiées (Ventoux, Lubéron, Sutlegen, Paphos, Sidi-Ifri) cessent leur photosynthèse entre -50 et -60 bars alors que 
dans le cas de Cedrus deodara, le niveau correspondant est de -30 bars. La comparaison de ces résultats avec d'autres travaux est limitée par le fait que la plupart des auteurs n'ont pas pris en compte le potentiel de base (PB), mais le potentiel hydrique au moment de la mesure de photosynthèse ( $\left({ }^{\prime}{ }^{\prime} N\right)$. Cependant, afin de situer le cèdre parmi les principales essences forestières, il est intéressant de remarquer que $\psi^{\prime}$ PN est, par nature, plus bas que le PB correspondant. Or, malgré ce décalage, on constate (tabl. 3) que, parmi les essences citées, le cèdre est celle dont l'assimilation photosynthétique peut se poursuivre aux niveaux de sécheresse les plus importants. Cette comparaison rapide est intéressante en première approche. Mais il sera nécessaire de l'améliorer par l'obtention d'informations similaires sur des espèces susceptibles de concurrencer le cèdre dans son aire d'introduction, notamment pins noirs et sapins méditerranéens.

De surcroît, il faut remarquer que la connaissance de la potentialité photosynthétique à un niveau de sécheresse donné doit être complétée par la prise en compte de la dynamique d'exploitation des réserves en eau du sol qui est spécifique de chaque essence.

Reçu pour publication en avril 1982.

\section{Summary}

Influence of a drought on the growth and photosynthesis of the genus Cedrus

The study of dryness thresholds influencing growth and photosynthesis was made for different provenances of the genus Cedrus. The results show significant differences for those droughts which affect the growth and photosynthesis.

The height growth is slower when the predawn potential reaches - 13 bars. It is stopped for potentials below -21 bars. In general the photosynthesis process is more resistant to a drought than the growth is. For the Cedrus atlantica (Ventoux) for example the photosynthesis still represents 55 p. 100 of its maximum maesured level when the growth stops. For the provenances which were studied (Ventoux, Luberon, Sidi-Ifri, Paphos, Sutlegen) the photosynthesis process stops when the predawn potential is between -50 and - 60 bars whereas that same level is - 30 bars in the case of Cedrus deodara.

Comparing these results with those obtained for other conifers, shows that the photosynthesis process of $C$. atlantica, $C$. libani and $C$. brevifolia are the least affected by a drought.

\section{Références bibliographiques}

Aussenac G., Granier A., 1978. - Quelques résultats de cinćtique journalière du potentiel de sève chez les arbres forestiers. Ann. Sci. for., 35 (1), 19-32.

Aussenac G., Granier A., Gross P., 1981. Etude de la croissance en hauteur du cèdre (Cedrus atlantica Manetti). Utilisation d'un apparcillage de mesure automatique. Ann. Sci. for., 38 (3), 301-316.

Aussenac G., Valette J.-C., 1982. Comportement estival de Cedrus atlantica Manetti, Quercus ilex L., Quercus pubescens Willd, et de divers pins dans le Mont Ventoux. Ann. Sci. for., 39 (1), 41-62.

BrIX H., 1979. Effects of plant water stress on photosynthesis and survival of four conifers. Can. J. for. Res., 9, 160-165. 
Finkle lostein D., 1981. Influence des conditions d’alimentation hydrique sur le débourrement et la croissince de jeunes plants de cèdres (Cedrus atlantica Manetti) cultivés en serre. Alnn. Sci. for., 38 (4), 109-126.

Finkfistein D., 1981. Contribution à la caractérisation écophysiologique du cèdre. Etude de la croissance et des échanges gazeux. Thèse troisième cycle, Nancy, 145 p.

Gi.likum C., Pirkpoint G., 1968. The influence of soil moisture deficits on seedlings growth of three coniferous species. Forest Chron., 44 (5), 26-29.

HiNCKI.EY T.M., Ritchit: G.A., 1979. A theoretical model for calculation of xylem sap pressure from climatological data. The American Midland Naturalist, 90 (1), 56-59.

El. Aoun M., 1980. Processus déterminant la production du pin d'Alep (P'inus halepensis Mill.), photosynthèse. croissance et répartition des assimilats. Thèse d'Etat ès Sciences Naturelles. Université Paris VII, 164 p.

PURITCH G.S., 1973. Effect of water stress on photosynthesis, respiration and transpiration on four Abies species. Can. J. for. Res., 3, 293-298.

StRANSKY J.J., Wilson D.R., 1964. Terminal elongation of loblolly and short leaf pine seedlings under soil moisture stress. Soil. Sci. Soc. of Am. Proceed., 28 (3), 439-449.

Watts W.R., NeIt.SON R.E., 1978. Photosynthesis in Sitka spruce (Picea sitchensis (Bong.) Carr.). VIII. - Measurements of stomatal conductance and $14 \mathrm{CO}_{2}$ uptake in controlled environments. $J$. appl. Ecol., 15, 245-255. 